\title{
Radioselenium in Duodenal Aspirate as an Assessment of Pancreatic Exocrine Function*
}

\author{
G. R. YOUNGS, J. E. AGNEW, G. E. LEVIN, I. A. D. BOUCHIER
}

British Medical fournal, 1971, 2, 252-255

\section{Summary}

A new test of pancreatic exocrine function is described in which radioselenium is measured in duodenal aspirate after an intravenous injection of ${ }^{75} \mathrm{Se}$-selenomethionine and administration of a Lundh test meal. Duodenal ${ }^{7}{ }^{5}$ Se activity gave good separation between normals and subjects with disease of the exocrine pancreas and correlated well with trypsin levels in the aspirate.

\section{Introduction}

Radioactive labelled amino-acids given intravenously to experimental animals are rapidly taken up by the acinar cells of the pancreas (Allfrey et al., 1953; Junquiera et al., 1955). Hansson (1959) gave intravenous ${ }^{35} \mathrm{~S}$-methionine to a variety of experimental animals and showed preferential uptake of the aminoacid by the pancreas, the radioactivity per gramme of tissue in this organ being six to eight times that in the next most active organ, the liver. After a delay of 45 to 60 minutes the isotope appeared in the protein fraction of the pancreatic juice attached to the pancreatic secretory enzymes.

It became possible to use this avidity of the pancreas for amino-acids for scintiscanning the human pancreas when Blau (1961) synthesized ${ }^{75} \mathrm{Se}$-selenomethionine, in which the central sulphur atom of methionine is replaced by a gamma-ray-emitting isotope of selenium. He showed that despite this modification to the molecule the ${ }^{75} \mathrm{Se}$-selenomethionine retained the physiological properties of methionine, including its preferential uptake by the pancreas (Blau and Manske, 1961). Since that time pancreatic scintiscanning has become a routine clinical investigation at many centres (Blau, 1964; Sodee, 1966; Brown et al., 1968; Melmed et al., 1968). There is no single ideal test for the diagnosis of pancreatic disease, and any test providing information on the function or morphology of this organ, in which it is notoriously difficult to detect the early lesion, is to be welcomed.

Although there have been a number of studies of selenomethionine metabolism in man (Awwad et al., 1966; Charlesworth et al., 1970), the appearance of the radioactive label in human duodenal juice after the intravenous injection of ${ }^{75} \mathrm{Se}$-selenomethionine has not been reported before. The present study investigates the appearance of ${ }^{75} \mathrm{Se}$ in duodenal test meal aspirate as a measure of pancreatic enzyme secretion and hence of pancreatic exocrine function. We believe that this test is a new approach to the diagnosis of pancreatic disease which can complement existing pancreatic function studies.

-A preliminary account of part of this work was presented at the annual meeting of the British Society of Gastroenterology, November 1970.

The Royal Free Hospital, London WE1X 8LF

G. R. YOUNGS, M.B., M.R.C.P., Registrar, Department of Medicine J. E. AGNEW, M.sc., Senior Physicist

G. E. LEVIN, M.B., M.SC., Lecturer, Department of Chemical Pathology I. A. D. BOUCHIER, M.D., F.R.C.P., Reader in Medicine

\section{Methods}

Duodenal levels of ${ }^{75} \mathrm{Se}$ and of trypsin were measured in 56 subjects-24 were classified as normal subjects, 20 had pancreatic disease, 3 had jaundice due to bile duct obstruction, and 9 had other miscellaneous diagnoses. The normal group consisted of patients undergoing investigation of abdominal pain for which no organic cause was subsequently found and of volunteers from whom fully informed consent was obtained. The subjects with disease of the pancreas included nine with carcinoma of the pancreas (eight proved at laparotomy) and seven with chronic pancreatitis (demonstrated at laparotomy in four and in the other cases by biochemical evidence of pancreatic steatorrhoea, abnormal glucose tolerance, and absent uptake on pancreatic scanning). Four subjects had relapsing pancreatitis (all with recurrent abdominal pain and raised serum amylase). The jaundice due to bile duct obstruction was shown at laparotomy to be due to a bile duct carcinoma in two subjects and to an ampullary stone in one. At operation the pancreas appeared normal in all three patients. Determinations of trypsin levels alone were carried out in six further subjects (four normal, one carcinoma of pancreas, and one chronic pancreatitis).

Pancreatic function was measured after stimulation with a Lundh test meal (Lundh, 1962; Cook et al., 1967) consisting of $18 \mathrm{~g}$ of corn oil, $15 \mathrm{~g}$ of Casilan, and $40 \mathrm{~g}$ of glucose made up to $300 \mathrm{ml}$ volume with water. The test was performed on the fasting subject intubated with a 12 French gauge Levin tube with five collecting holes in the last $6 \mathrm{~cm}$ of the tube. A mercury bag was attached to the end of the tube, which was screened under $x$-ray control to lie at or just beyond the duodenal-jejunal flexure. The patient then drank the test meal, and 10 minutes later ${ }^{75} \mathrm{Se}-$ selenomethionine was given intravenously. Two dosage schedules were used: $3 \mu \mathrm{Ci} / \mathrm{kg}$ body weight to 47 patients receiving a simultaneous pancreatic scan and $0.3 \mu \mathrm{Ci} / \mathrm{kg}$ body weight to nine patients not being scanned. The normal volunteers received only the smaller dose. The duodenal contents were collected by continuous siphonage and intermittent aspiration for up to 150 minutes in four or five separate 30-minute periods. At the end of the test a gastric sample was collected on withdrawal of the tube. The appearance and $\mathrm{pH}$ of the aspirate were monitored during the test, and if the aspirate was bile free or acid the position of the tube was checked at the end of the test by $x$-ray examination. The duodenal aspirate was collected on ice and then stored at $-20^{\circ} \mathrm{C}$. The juice was analysed for ${ }^{75} \mathrm{Se}$ activity by means of a Packard Auto-Gamma Spectrometer.

Trypsin was assayed according to the method of Hummel (1959) by measuring the rate of hydrolysis of $p$-toluene sulphonyl-L-arginine methyl ester. Results were expressed as $\mu \mathrm{mol}$ hydrolysed per minute per $\mathrm{ml}$ of duodenal aspirate at $22^{\circ} \mathrm{C}$.

${ }^{35} \mathrm{Se}$ activity was measured in the gastric sample collected at the end of each test. In four of the normal subjects specimens of gastric juice were taken at 30-minute intervals for 150 minutes and the ${ }^{75} \mathrm{Se}$ activity was measured. Radioactivity in human bile was measured in separate experiments in two patients with $\mathrm{T}$-tubes in the common bile duct, one patient having had a biliary stricture and the other a stone impacted in the ampulla of Vater. Bile was collected for three hours following intravenous selenomethionine $(0.3 \mu \mathrm{Ci} / \mathrm{kg})$. No test meal was given to either patient. 


\section{Results}

The results of the Lundh test are shown in Fig. 1. Samples from each 30-minute period in the first two hours of collection were pooled and the result was expressed as the mean tryptic activity for the two-hour collection period. The distribution in the normal group was shown to be log normal and the range was calculated from the mean \pm 2 S.D. after a logarithmic transformation. The range thus obtained was found to be 26 to $73 \mu \mathrm{mol} / \mathrm{ml} / \mathrm{min}$, with a mean activity of $44 \mu \mathrm{mol} / \mathrm{ml} / \mathrm{min}$. The mean tryptic activity was significantly different from normal in subjects suffering from chronic pancreatitis and carcinoma of the pancreas, only one of these subjects giving a result within the normal range. This is in keeping with published results. The values for subjects with relapsing pancreatitis were more variable. The values of the three subjects with jaundice due to bile duct obstruction were within the normal range.

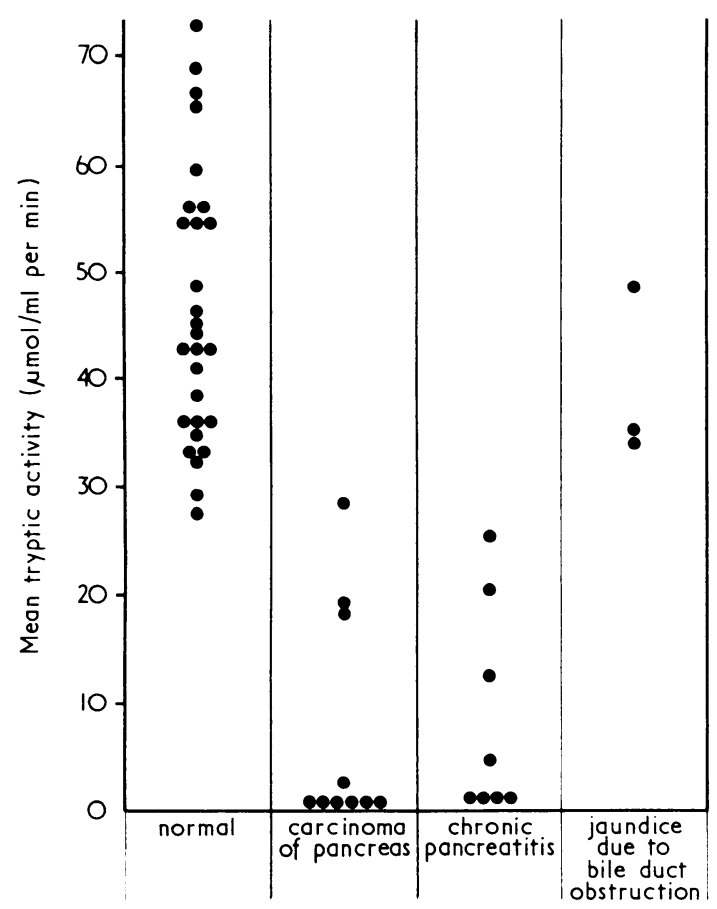

FIG. 1-Results of Lundh test: mean tryptic activitics.

The mean ${ }^{i 5} \mathrm{Se}$ activities in duodenal and gastric aspirates from normal subjects are shown in Fig. 2. The ${ }^{75} \mathrm{Se}$ activity is expressed as a percentage of the dose injected per $100 \mathrm{ml}$ of aspirate. The vertical lines on the figure indicate the approximate $95 \%$ confidence limits for the mean values (arithmetic mean \pm 2 standard errors of the mean) not the range of individual values. In the first hour the ${ }^{75} \mathrm{Se}$ activity in the duodenal juice of normal subjects remained low but then rose rapidly and was still rising at 150 minutes. The line obtained in those subjects receiving the larger dose of selenomethionine was almost identical to that obtained in those receiving the smaller dose, and the line shown in Fig. 2 represents both groups. In contrast the ${ }^{75} \mathrm{Se}$ activity in normal gastric juice remained low throughout the test except in one patient in whom an anomalously high level of $0 \cdot 17 \% / 100 \mathrm{ml}$ was reached in the 90 to 120 -minute specimen. For comparison Fig. 2 also shows the mean lcvel of ${ }^{75} \mathrm{Se}$ in samples of bile obtained from the two patients with indwelling T-tubes. The ${ }^{75} \mathrm{Se}$ levels in both patients remained low throughout the period of collection.

At the end of the test there was a sixfold difference between the ${ }^{75} \mathrm{Se}$ activity in the duodenal aspirate and in the gastric juice or bile.

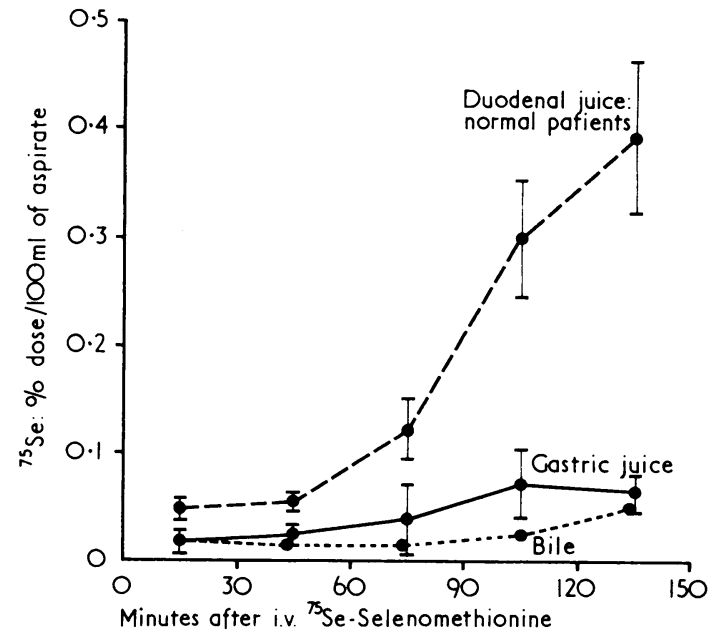

FIG. 2-Mean ".5e levels in normal duodenal and gastric aspirates and in bile: variation with time. Vertical lines indicate \pm 2 S.E. of mean.

The ise in the duodenal aspirate from subjects with carcinoma of the pancreas and chronic pancreatitis rose only slowly during the test (Fig. 3). In the specimen collected 120 to 150 minutes after injection of the selenomethionine there was a fourfold difference between the mean ${ }^{75}$ Se activity in the normal group of subjects compared with both groups of subjects with confirmed disease of the exocrine pancreas.

The radioactivity levels in the 90 to 120 -minute specimen in normal and abnormal subjects are shown in Fig. 4. Reasonable separation was obtained between the normal group and those with chronic pancreatitis and carcinoma, the distribution being comparable to that with the Lundh test (Fig. 1). As with the trypsin levels, the radioselenium levels in the normal subjects appeared to show a log normal distribution. The normal range at 90 to 120 minutes-calculated from the mean \pm 2 S.D. after a logarithmic transformation-was found to be $0 \cdot 12-0.66 \%$ of the dose per $100 \mathrm{ml}$ of duodenal aspirate. For comparing normal and abnormal levels the normal range at this time was chosen in preference to that at 120 to 150 minutes as only about half of the subjects had collections up to 150 minutes.

Among the patients with carcinoma whose ${ }^{75} \mathrm{Se}$ results are shown in Fig. 4, the highest ${ }^{75}$ Se level was found in the only subject with a secondary pancreatic carcinoma. The other two patients with comparatively high ${ }^{75}$ Se levels $(0.12$ and $0 \cdot 10 \% /$ $100 \mathrm{ml}$ respectively) also had comparatively high mean trypsin

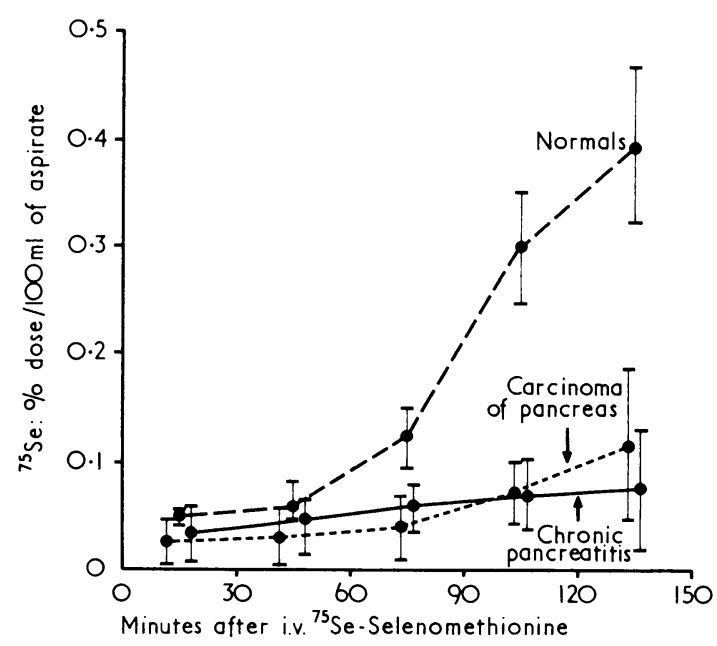

FIG. 3-Mean duodenal ${ }^{75} \mathrm{Se}$ levels in pancreatic disease. Vertical lines indicate \pm 2 S.E. of mean. 


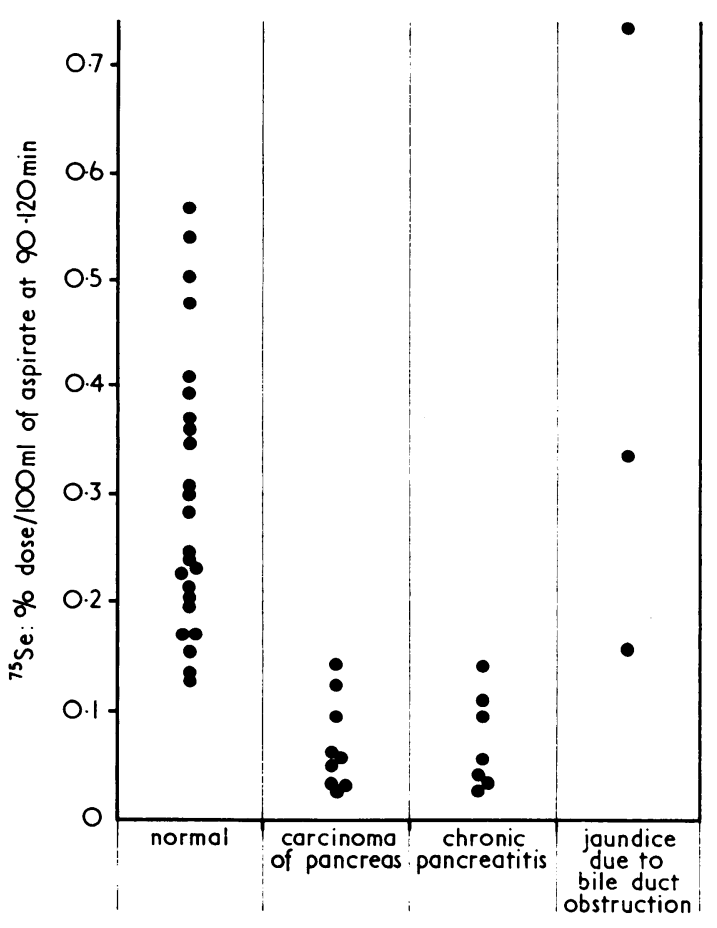

FIG. 4-Duodenal "isc activity at 90 to 120 minutes.

levels (29 and $19 \mu \mathrm{mol} / \mathrm{ml} / \mathrm{min}$ ) and areas of functioning pancreatic tissue shown on the pancreatic scan. The is Se levels reached in the patient with the ampullary stone and in one patient with bile duct carcinoma fell within the normal range. The level in the second patient with bile duct carcinoma was above the normal range. The values in patients with relapsing pancreatitis were again variable.

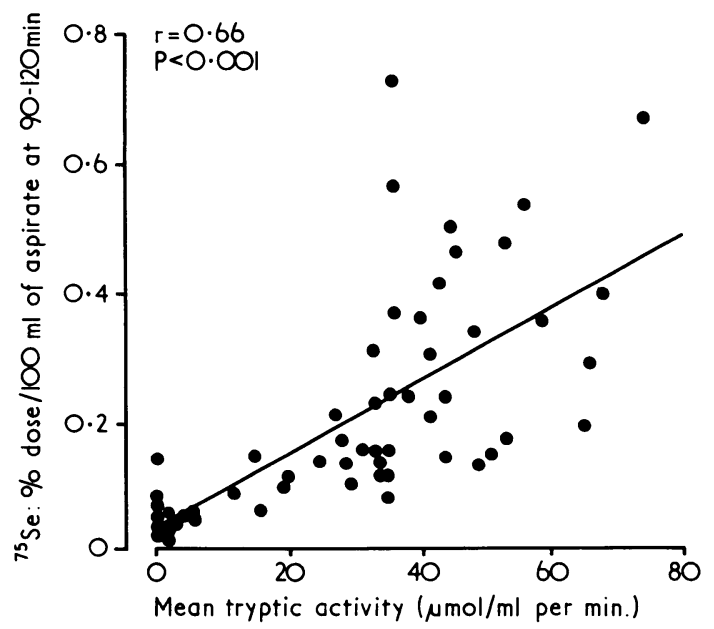

FIG. 5-Comparison betwecn duodenal ${ }^{75} \mathrm{Sc}$ activitics at 90 to 120 minutes and mean tryptic activities.

For all subjects studied the ${ }^{75} \mathrm{Se}$ activity reached in the 90 to 120-minute specimen was compared with the mean tryptic activity of the aspirate (Fig. 5). The coefficient of correlation was $0.66(P<0.001)$. The correlation between ${ }^{75} \mathrm{Se}$ and the peak tryptic activity reached in any one 30-minute collection period was $0.75(P<0.001)$. In the subjects in whom the collections were made for 150 minutes similar correlation was obtained: 0.77 for the mean and 0.75 for the peak tryptic activity $(\mathrm{P}<$ 0.001 ).
Preliminary experiments on duodenal juice uncontaminated by test meal, obtained after stimulation of the pancreas with intravenous secretin and pancreozymin, showed that the protein fraction of the juice, precipitated with trichloracetic acid, contained about balf the ${ }^{75} \mathrm{Se}$ activity. Cellulose acetate electrophoresis of the juice from one subject was caried out. On staining the strips for protein with nigrosin, six to eight bands were seen and these appeared to correspond with the bands shown to be pancreatic enzymes by Silberberg and Hadorn (1968). Staining for trypsin was positive and it was possible to demonstrate significant amounts of radioselenium activity in five of these bands.

\section{Discussion}

The good separation given by the radioselenium test between normals and subjects with confirmed pancreatic disease and the good correlation between the radioselenium and tryptic activities suggest that the test may be useful as a measure of pancreatic enzyme secretion and hence of pancreatic exocrine function. This view is supported by the finding that a significant proportion of the ${ }^{75} \mathrm{Se}$ appearing in the duodenal aspirate is attached to the protein fraction, and the electrophoresis experiment suggests that the radioselenium is labelled to several of the pancreatic enzymes.

Animal experiments indicate that the time taken to reach the peak ${ }^{75} \mathrm{Se}$ activity in pancreatic juice lies between 120 and 240 minutes (Hansson, 1959; Hansson and Blau, 1963; Goidsenhoven et al., 1967). We have not yet determined the timing of peak radioactivity in the duodenal aspirate in man; nevertheless, our results indicate that the measurement of duodenal radioactivity at 120 or 150 minutes after the injection of ${ }^{75} \mathrm{Se}$-selenomethionine correlates significantly with duodenal tryptic activity and that the agreement between these two measurements was poor in very few subjects.

Two hours after an intravenous injection of labelled methionine about $85 \%$ of the radioactivity lies in the protein fraction of pancreatic juice obtained by cannulation of the pancreatic duct in the cat and dog (Hansson, 1959; Goidsenhoven et al., 1967). Our preliminary experiments showing that about half the radioactivity lies in the protein fraction of the duodenal juice at 120 minutes suggest that not all the radioactivity of the duodenal aspirate originates from the pancreas. Fig. 2 shows that there is little appreciable activity in gastric juice or bile, and therefore it is possible that the non-protein ${ }^{75} \mathrm{Se}$ activity may originate from an amino-acid flux across the mucous membrane of the small intestine (Spencer and Blau, 1962).

In similar experiments on four subjects with T-tubes, Charlesworth et al. (1970) showed that the ${ }^{75} \mathrm{Se}$ activity in bile is not protein bound. In their patients the bile level of ${ }^{75} \mathrm{Se}$ reached a maximum at 10 minutes and then gradually fell over a two-hour period. Thus though biliary ${ }^{75} \mathrm{Se}$ may contribute to the nonprotein bound ${ }^{75} \mathrm{Se}$ fraction in the duodenal aspirate, it is unlikely to be the cause of the rise in duodenal radioactivity in normal subjects (Figs. 2 and 3). The experiments on which the biliary ${ }^{75}$ Se levels shown in Fig. 2 are based were not, however, physiological in that before the T-tube insertion both patients had suffered a period of obstructive jaundice and at the time of the experiment there had been considerable depletion of the bile salt pool because of continuous biliary drainage.

The contribution of biliary ${ }^{75} \mathrm{Se}$ to the duodenal radioactivity must also depend on the unknown relative volumes of bile, pancreatic, and other secretions emptying into the duodenum during digestion. There was no indication that bile volume increased during the test as colouring of the aspirate due to bile pigments tended to remain constant and total bile acids, measured in the aspirate of five of the normal subjects, also remained constant once gall bladder emptying had occurred. In the three subjects in whom owing to bile duct obstruction there could not have been any significant flow of biliary ${ }^{75} \mathrm{Se}$ into the duodenum the duodenal radioactivity was normal or above normal. 
Given that duodenal ${ }^{75} \mathrm{Se}$ originates mainly from the pancreas, low selenium levels should be expected in patients with disease of the exocrine pancreas. Eight out of 10 patients with carcinoma of the pancreas and five out of eight with chronic pancreatitis had zero trypsin levels, and in these patients the ${ }^{75} \mathrm{Se}$ levels failed to rise, radioactivity at 90 to 120 minutes being no higher than levels found in gastric aspirate. The position of the Levin tube at the end of the test was checked by $x$-ray examination of all these patients to exclude the possibility that the tube had refluxed into the stomach. Two patients with cancer of the body of the pancreas and three with chronic pancreatitis had appreciable, though subnormal, tryptic activity and the rdioactivity was also higher than basal levels though still subnormal in all but one patient who reached a low normal level. Eight of the 10 subjects with carcinoma of the pancreas had obstructive jaundice and bile-free duodenal aspirates. None of the patients with chronic pancreatitis was jaundiced and in all eight the duodenal aspirate contained bile. The similarity of the duodenal ${ }^{75} \mathrm{Se}$ levels in these two groups of patients provides further evidence that the ${ }^{75} \mathrm{Se}$ activities of the duodenal juice are independent of the presence of bile in the aspirate.

The radioselenium test described in this paper is no more discriminatory than the Lundh test, as can be seen by comparing Figs. 1 and 4. It is possible that the discriminatory value of the radioselenium test might be improved by measuring the ${ }^{75} \mathrm{Se}$ when it reaches its peak concentration in the aspirate but this would prolong the duration of the test, and, in addition, the volume of aspirate diminishes considerably after two hours.

\section{ADVANTAGES}

There are a number of advantages to the radioselenium test of pancreatic function. Firstly, the likelihood that the label is incorporated into all the pancreatic secretory enzymes and thus the label is a measure of total enzyme output and not just a single enzyme. It has been shown that measurement of the protein concentration in pancreatic juice of cattle (Keller et al., 1958) and dogs (Cooke et al., 1967) reflects pancreatic enzyme secretion, but such a measurement would not be applicable to the Lundh test as the test meal contains protein. Secondly, continuous collections of duodenal juice are not necessary as a single specimen at 120 or 150 minutes will suffice. Thirdly, in some centres it may be simpler to measure duodenal ${ }^{75} \mathrm{Se}$ activity than to measure trypsin. Fourthly, the half-life of ${ }^{75} \mathrm{Se}$ is 120 days, so the specimens are easily stored and transmitted. Fifthly, the test is effective when using one-tenth of the dose of selenomethionine normally used for pancreatic scanning and this is of advantage in the younger patients. The cost of the smaller dose is about $£ 1$.
An ideal and comprehensive test of pancreatic function has yet to be devised, and it is unlikely that even a perfect function test could invariably distinguish between the normal and abnormal pancreas for, as with lung function tests or blood pressure readings, the range of normal is wide and indefinite. Moreover, any test involving intubation may alter the delicate physiological balance between the several organs involved. In this study the collection of duodenal aspirate for trypsin and ${ }^{75} \mathrm{Se}$ estimations was performed simultaneously with conventional and subtraction pancreas scans and a liver scan. Such a combined study took about three hours to complete. The value of such a combined diagnostic approach is at present being assessed.

We wish to thank Miss R. Lydford and Miss C. Taylor for their skilled technical help and Miss E. Bateman for preparation of the Lundh test meals. We are grateful to Miss Pamela Carlsson for the electrophoretic studies and to Dr. Fernando Portella for measurement of the bile acids. Professor D. N. Baron and Mr. H. S. Williams provided valuable criticism of the manuscript. We are grateful to Dr. H. S. Wiggins for advice on performing the Lundh test.

\section{References}

Allfrey, V., Daley, M. M., and Mirsky, A. E. (1953). Fournal of General Physiology, 37, 157.

Awwad, H. K., Potchen, E. J., Adelstein, S. J., and Dealy, J. B. (1966). Metabolism, 15, 626.

Blau, M. (1961). Biochimica et Biophysica Acta, 49, 389.

Blau, M., and Manske, R. F. (1961). Fournal of Nuclear Medicine, 2, 102.

Blau, M. (1964). In Medical Radioisotope Scanning, vol. 2, p. 275 . Vienna International Atomic Energy Agency.

Brown, P. W., et al. (1968). Lancet, 1, 160 .

Charlesworth, D., Testa, H. J., Pullan, B. R., and Torrance, H. B. (1970), British fournal of Surgery, $57,413$.

Cook, B., Lennard-Jones, J. E., Sherif, S. M., and Wiggins, H. S. (1967) Gut, 8, 408.

Cooke, A. R., Nahrwold, D. L., and Grossman, M. I. (1967). American fournal of Physiology, 213, 637 .

Goidsenhoven, G. E. van, Denk, A. F., Pfleger, B. A., and Knight, W. A (1967). Gastroenterology, 53, 403.

Hansson, E. (1959). Acta Physiologica Scandinavica, 46, Suppl. No. 161, p. 1 Hansson, E., and Blau, M. (1963). Biochemical and Biophysical Research Communications, 13, 71 .

Hummel, B. C. W. (1959). Canadian Fournal of Biochemistry and Physiology, 37, 393. Junquiera, L. C. U., Hirsch, G. C., and Rothschild, H. A. (1955). Biochemical

Keller, P. J., Cohen, E., and Neurath, H. (1958). Fournal of Biological Chemistry, $233,344$.

Lundh, G. (1962). Gastroenterology, 42, 275.

Melmed, R. N., Agnew, J. E., and Bouchier, I. A. D. (1968). Quarterly Fournal of Medicine, 37, 607.

Silberberg, V. L., and Hadorn, B. (1968). Biochimica et Biophysica Acta, 167,616 .

Sodee, D. B. (1966). Radiology, 87, 641

Spencer, R. P., and Blau, M. (1962). Science, 136, 155. 